\title{
A SURVEY OF WALL'S FINITENESS OBSTRUCTION
}

\author{
STEVE FERRY AND ANDREW RANICKI
}

\section{Contents}

Introduction

1. Finite domination

2. The projective class group $K_{0}$

3. The finiteness obstruction

4. The topological space-form problem

5. The Siebenmann end obstruction

6. Connections with Whitehead torsion

7. The splitting obstruction

8. The triangulation of manifolds References

\section{INTRODUCTION}

Wall's finiteness obstruction is an algebraic $K$-theory invariant which decides if a finitely dominated space is homotopy equivalent to a finite $C W$ complex. The invariant was originally formulated in the context of surgery on $C W$ complexes, generalizing Swan's application of algebraic $K$-theory to the study of free actions of finite groups on spheres. In the context of surgery on manifolds, the invariant first arose as the Siebenmann obstruction to closing a tame end of a non-compact manifold. The object of this survey is to describe the Wall finiteness obstruction and some of its many applications to the surgery classification of manifolds. The book of Varadarajan [38 and the survey of Mislin [24] deal with the finiteness obstruction from a more homotopy theoretic point of view.

\section{Finite domination}

A space is finitely dominated if it is a homotopy retract of a finite complex. More formally:

Definition 1.1. A topological space $X$ is finitely dominated if there exists a finite $C W$ complex $K$ with maps $d: K \rightarrow X, s: X \rightarrow K$ and a homotopy

$$
d \circ s \simeq \operatorname{id}_{X}: X \rightarrow X .
$$


Example 1.2. (i) A compact $A N R X$ is finitely dominated (Borsuk [3]). In fact, a finite dimensional $A N R X$ can be embedded in $\mathbb{R}^{N}$ ( $N$ large), and $X$ is a retract of an open neighbourhood $U \subset \mathbb{R}^{N}$ - there exist a retraction $r: U \rightarrow X$ and a compact polyhedron $K \subset U$ such that $X \subset K$, so that the restriction $d=r \mid: K \rightarrow X$ and the inclusion $s: X \rightarrow K$ are such that $d \circ s=\operatorname{id}_{X}: X \rightarrow X$.

(ii) A compact topological manifold is a compact $A N R$, and hence finitely dominated.

The problem of deciding if a compact $A N R$ is homotopy equivalent to a finite $C W$ complex was first formulated by Borsuk [4]. (The problem was solved affirmatively for manifolds by Kirby and Siebenmann in 1969, and in general by West in 1974 - see section 8 below.) The problem of deciding if a finitely dominated space is homotopy equivalent to a finite $C W$ complex was first formulated by J.H.C.Whitehead. Milnor 23 remarked: "It would be interesting to ask if every space which is dominated by a finite complex actually has the homotopy type of a finite complex. This is true in the simply connected case, but seems like a difficult problem in general."

Here is a useful criterion for recognizing finite domination:

Proposition 1.3. $A C W$ complex $X$ is finitely dominated if and only if there is a homotopy $h_{t}: X \rightarrow X$ such that $h_{0}=i d$ and $h_{1}(X)$ has compact closure.

Proof. If $d: K \rightarrow X$ is a finite domination with right inverse $s$, let $h_{t}$ be a homotopy from the identity to $d \circ s$. Since $h_{1}(X) \subset d(K)$, the closure of $h_{1}(X)$ is compact in $X$. Conversely, if the closure of $h_{1}(X)$ is compact in $X$, let $K$ be a finite subcomplex of $X$ containing $h_{1}(X)$. Setting $d$ equal to the inclusion $K \rightarrow X$ and $s$ equal to $h_{1}: X \rightarrow K$ shows that $X$ is finitely dominated.

It is possible to relate finitely dominated spaces, finitely dominated $C W$ complexes and spaces of the homotopy type of $C W$ complexes, as follows.

Proposition 1.4. (i) A finitely dominated topological space $X$ is homotopy equivalent to a countable $C W$ complex.

(ii) If $X$ is homotopy dominated by a finite $k$-dimensional $C W$ complex, then $X$ is homotopy equivalent to a countable $(k+1)$-dimensional $C W$ complex.

Proof. The key result is the trick of Mather [22, which shows that if $d$ : $K \rightarrow X, s: X \rightarrow K$ are maps such that $d \circ s \simeq \operatorname{id}_{X}: X \rightarrow X$ then $X$ is homotopy equivalent to the mapping telescope of $s \circ d: K \rightarrow K$. This requires the calculus of mapping cylinders, which we now recall.

By definition, the mapping cylinder of a map $f: K \rightarrow L$ is the identification space

$$
M(f)=(K \times[0,1] \cup L) /((x, 1) \sim f(x)) .
$$


We shall use three general facts about mapping cylinders:

- If $f: K \rightarrow L$ and $g: L \rightarrow M$ are maps and $k: K \rightarrow M$ is homotopic to $g \circ f$, the mapping cylinder $M(k)$ is homotopy equivalent rel $K \cup M$ to the concatenation of the mapping cylinders $M(f)$ and $M(g)$ rel $K \cup M$.

- If $f, g: K \rightarrow L$ with $f \sim g$, then the mapping cylinder of $f$ is homotopy equivalent to the mapping cylinder of $g$ rel $K \cup L$.

- Every mapping cylinder is homotopy equivalent to its base rel the base.

The mapping telescope of a map $\alpha: K \rightarrow K$ is the countable union

$$
\bigcup_{i=0}^{\infty} M(\alpha)=\bigcup_{i=0}^{\infty} K \times[i, i+1] /\{(x, i) \sim(\alpha(x), i+1)\} .
$$

For any maps $d: K \rightarrow X, s: X \rightarrow K$ we have

$$
\bigcup_{i=0}^{\infty} M(d \circ s)=X \times I \cup \bigcup_{i=0}^{\infty} M(s \circ d)
$$

with $\bigcup_{i=0}^{\infty} M(s \circ d)$ a deformation retract, so that

$$
\bigcup_{i=0}^{\infty} M(d \circ s) \simeq \bigcup_{i=0}^{\infty} M(s \circ d)
$$

To see why this holds, note that $\bigcup_{i=0}^{\infty} M(d \circ s)$ is homotopy equivalent to an infinite concatenation of alternating $M(d)$ 's and $M(s)$ 's which can also be thought of as an infinite concatenation of $M(s)$ 's and $M(d)$ 's. Essentially, we're reassociating an infinite product. Here is a picture of this part of the construction.

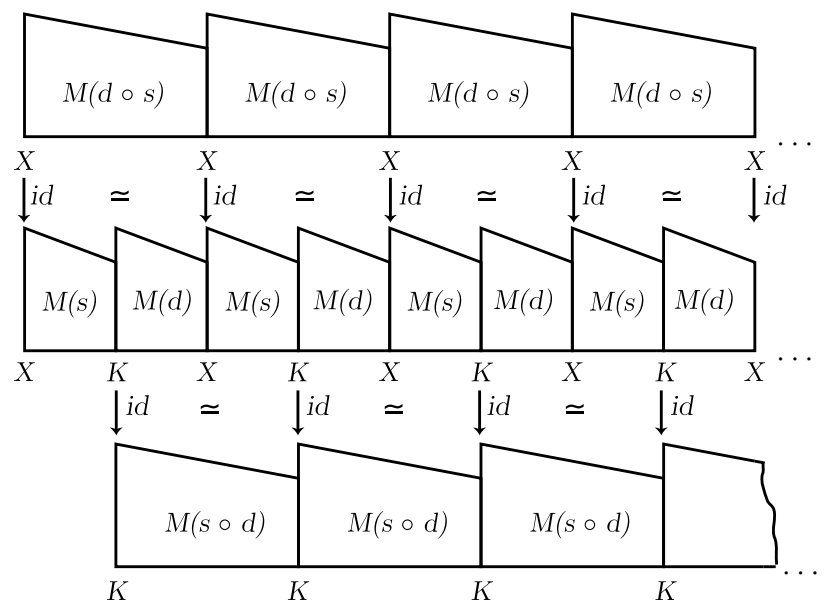


(i) If $d: K \rightarrow X, s: X \rightarrow K$ are such that $d \circ s \simeq \operatorname{id}_{X}: X \rightarrow X$ there is defined a homotopy idempotent of a finite $C W$ complex

$$
\alpha=s \circ d: K \rightarrow K
$$

with $\alpha \circ \alpha \simeq \alpha: K \rightarrow K$. We have homotopy equivalences

$$
X \simeq X \times[0, \infty) \simeq \bigcup_{i=0}^{\infty} M\left(\operatorname{id}_{X}\right) \simeq \bigcup_{i=0}^{\infty} M(d \circ s) \simeq \bigcup_{i=0}^{\infty} M(s \circ d)=\bigcup_{i=0}^{\infty} M(\alpha) .
$$

The mapping telescope $\bigcup_{i=0}^{\infty} M(\alpha)$ is a countable $C W$ complex.

(ii) As for (i), but with $K k$-dimensional.

This proposition is comforting because it shows that the finiteness problem for arbitrary topological spaces reduces to the finiteness problem for $C W$ complexes. One useful consequence of this is that we can use the usual machinery of algebraic topology, including the Hurewicz and Whitehead theorems, to detect homotopy equivalences.

Proposition 1.5. (Mather [22]) A topological space $X$ is finitely dominated if and only if $X \times S^{1}$ is homotopy equivalent to a finite $C W$ complex.

Proof. The mapping torus of a map $\alpha: K \rightarrow K$ is defined (as usual) by

$$
T(\alpha)=(K \times[0,1]) /\{(x, 0) \sim(\alpha(x), 1)\} .
$$

For any maps $d: K \rightarrow X, s: X \rightarrow K$ there is defined a homotopy equivalence

$$
T(d \circ s: X \rightarrow X) \rightarrow T(s \circ d: K \rightarrow K) ;(x, t) \mapsto(s(x), t) .
$$

If $d \circ s \simeq \operatorname{id}_{X}: X \rightarrow X$ and $K$ is a finite $C W$ complex we thus have homotopy equivalences

$$
X \times S^{1} \simeq T\left(\operatorname{id}_{X}\right) \simeq T(s \circ d)
$$

with $T(s \circ d)$ a finite $C W$ complex.

Conversely, if $X \times S^{1}$ is homotopy equivalent to a finite $C W$ complex $K$ then the maps

$$
d: K \simeq X \times S^{1} \stackrel{\text { proj. }}{\longrightarrow} X, s: X \stackrel{\text { incl. }}{\longrightarrow} X \times S^{1} \simeq K
$$

are such that $d \circ s \simeq \operatorname{id}_{X}$, and $X$ is dominated by $K$.

\section{The Projective Class group $K_{0}$}

Let $\Lambda$ be a ring (associative, with 1 ).

Definition 2.1. A $\Lambda$-module $P$ is $f . g$. projective if it is a direct summand of a f.g. (= finitely generated) free $\Lambda$-module $\Lambda^{n}$, with $P \oplus Q=\Lambda^{n}$ for some direct complement $Q$. 
A $\Lambda$-module $P$ is f. g. projective if and only if $P$ is isomorphic to $\operatorname{im}(p)$ for some projection $p=p^{2}: \Lambda^{n} \rightarrow \Lambda^{n}$.

Definition 2.2. (i) The projective class group $K_{0}(\Lambda)$ is the Grothendieck group of stable isomorphism classes of $\mathrm{f}$. g. projective $\Lambda$-modules.

(ii) The reduced projective class group $\widetilde{K}_{0}(\Lambda)$ is the quotient of $K_{0}(\Lambda)$ by the subgroup generated by formal differences $\left[\Lambda^{m}\right]-\left[\Lambda^{n}\right]$ of f. g. free modules.

Thus an element of $\widetilde{K}_{0}(\Lambda)$ is an equivalence class $[P]$ of f. g. projective $\Lambda$-modules, with $\left[P_{1}\right]=\left[P_{2}\right]$ if and only if there are f.g. free $\Lambda$-modules $F_{1}$ and $F_{2}$ so that $P_{1} \oplus F_{1}$ is isomorphic to $P_{2} \oplus F_{2}$. In particular, $[P]$ is trivial if and only if $P$ is stably free, that is, if there is a f.g. free module $F$ so that $P \oplus F$ is free.

Chapter 1 of Rosenberg 35 is a general introduction to the projective class groups $K_{0}(\Lambda), \widetilde{K}_{0}(\Lambda)$ and their applications, including the Wall finiteness obstruction.

Example 2.3. There are many groups $\pi$ for which

$$
\widetilde{K}_{0}(\mathbb{Z}[\pi])=0,
$$

including virtually polycyclic groups, a class which includes free and free abelian groups.

At present, no example is known of a torsion-free infinite group $\pi$ with $\widetilde{K}_{0}(\mathbb{Z}[\pi]) \neq 0$. Indeed, Hsiang has conjectured that $\widetilde{K}_{0}(\mathbb{Z}[\pi])=0$ for any torsion-free group $\pi$. (See Farrell and Jones [11, pp. 9-11). On the other hand:

Example 2.4. (i) There are many finite groups $\pi$ for which

$$
\widetilde{K}_{0}(\mathbb{Z}[\pi]) \neq 0
$$

including the cyclic group $\mathbb{Z}_{23}$.

(ii) The reduced projective class group of the quaternion group

$$
Q(8)=\{ \pm 1, \pm i, \pm j, \pm k\}
$$

is the cyclic group with 2 elements

$$
\widetilde{K}_{0}(\mathbb{Z}[Q(8)])=\mathbb{Z}_{2},
$$

generated by the f. g. projective $\mathbb{Z}[Q(8)]$-module

$$
P=\operatorname{im}\left(\left(\begin{array}{cc}
1-8 N & 21 N \\
-3 N & 8 N
\end{array}\right): \mathbb{Z}[Q(8)] \oplus \mathbb{Z}[Q(8)] \rightarrow \mathbb{Z}[Q(8)] \oplus \mathbb{Z}[Q(8)]\right)
$$

with $N=\sum_{g \in Q(8)} g$. 
We refer to Oliver [25] for a survey of the computations of $\widetilde{K}_{0}(\mathbb{Z}[\pi])$ for finite groups $\pi$.

\section{The FINITENESS OBSTRUCTION}

Here is the statement of Wall's theorem.

Theorem 3.1. ([39, [40]) (i) A finitely dominated space $X$ has a finiteness obstruction

$$
[X] \in \widetilde{K}_{0}\left(\mathbb{Z}\left[\pi_{1}(X)\right]\right)
$$

such that $[X]=0$ if and only if $X$ is homotopy equivalent to a finite $C W$ complex.

(ii) If $\pi$ is a finitely presented group then every element $\sigma \in \widetilde{K}_{0}(\mathbb{Z}[\pi])$ is the finiteness obstruction of a finitely dominated $C W$ complex $X$ with $[X]=\sigma$, $\pi_{1}(X)=\pi$.

(iii) A CW complex $X$ is finitely dominated if and only if $\pi_{1}(X)$ is finitely presented and the cellular $\mathbb{Z}\left[\pi_{1}(X)\right]$-module chain complex $C_{*}(\widetilde{X})$ of the universal cover $\widetilde{X}$ is chain homotopy equivalent to a finite chain complex $\mathcal{P}$ of f.g. projective $\mathbb{Z}\left[\pi_{1}(X)\right]$-modules.

Outline of proof (i) Here is an extremely condensed sketch of Wall's argument from [39]. If $d: K \rightarrow X$ is a finite domination with $X$ a $C W$ complex, we can assume that $d$ is an inclusion by replacing $X$, if necessary, by the mapping cylinder of $d$. For each $\ell \geq 2$, we then have a split short exact sequence of abelian groups

$$
0 \rightarrow \pi_{\ell+1}(X, K) \rightarrow \pi_{\ell}(K) \rightarrow \pi_{\ell}(X) \rightarrow 0 .
$$

Wall gives a special argument to show that $d$ can be taken to induce an isomorphism on $\pi_{1}$ and then shows that $\pi_{\ell+1}(X, K)$ is f. g. as a module over $\mathbb{Z}\left[\pi_{1}(X)\right]$, provided that $\pi_{q}(X, K)=0$ for $q \leq \ell, \ell \geq 2$. This allows him to attach $\ell+1$-cells to form a complex $\bar{K} \supset K$ and a map $\bar{d}: \bar{K} \rightarrow X$ extending $d$ so that $\bar{d}$ induces isomorphisms on homotopy groups through dimension $\ell$. Since $\bar{d}$ is a domination with the same right inverse $s$, this process can be repeated. In the case $\ell \geq \operatorname{dim}(K)$, Wall shows that $\pi_{\ell+1}(X, K)$ is a f. $g$. projective module over $\mathbb{Z}\left[\pi_{1}(X)\right]$. If $\pi_{\ell+1}(X, K)$ is free (or even stably free) we can attach $\ell+1$-cells to kill $\pi_{\ell+1}(X, K)$ without creating new problems in higher dimensions. The result is that $\bar{d}$ is a homotopy equivalence from $\bar{K}$ to $X$. If this module is not stably free, we are stuck and the finiteness obstruction is defined to be

$$
[X]=(-1)^{\ell+1}\left[\pi_{\ell+1}(X, K)\right] \in \widetilde{K}_{0}\left(\mathbb{Z}\left[\pi_{1}(X)\right]\right) .
$$

(ii) Given a finite $C W$ complex $K$ and a nontrivial $\sigma \in \widetilde{K}_{0}\left(\mathbb{Z}\left[\pi_{1}(K)\right]\right)$, here is one way to construct a $C W$ complex with finiteness obstruction $\pm \sigma$ : let $\sigma$ be represented by a f. g. projective module $P$ and let $F=P \oplus Q$ be free 
of rank $n$. Let $A$ be the matrix of the projection $p: F \rightarrow P \rightarrow F$ with respect to a standard basis for $F$. Now let

$$
L=K \vee \bigvee_{i=1}^{n} S_{i}^{\ell}
$$

There is a split short exact sequence

$$
0 \longrightarrow \pi_{\ell}(K) \stackrel{r_{*}}{\stackrel{i_{*}}{\longrightarrow}} \pi_{\ell}(L) \longrightarrow \pi_{\ell}(L, K) \longrightarrow 0,
$$

where $r: L \rightarrow K$ is the retraction which sends the $S^{\ell}$ 's to the basepoint. Since

$$
\pi_{\ell}(L, K) \cong \pi_{\ell}(\tilde{L}, \tilde{K}) \cong H_{\ell}(\tilde{L}, \tilde{K}) \cong F,
$$

we can define $\alpha: L \rightarrow L$ so that $\alpha \mid K=i d$ and so that $\alpha_{*}: \pi_{\ell}(L) \rightarrow \pi_{\ell}(L)$ has the matrix

$$
\left(\begin{array}{cc}
i d & 0 \\
0 & A
\end{array}\right)
$$

with respect to the direct sum decomposition $\pi_{\ell}(L) \cong \pi_{\ell}(K) \oplus F$. Since $A^{2}=A$, it is easy to check that $\alpha$ is homotopy idempotent, i.e. that $\alpha \circ \alpha \sim \alpha$ rel $K$.

Let $X$ be the infinite direct mapping telescope of $\alpha$ pictured below.

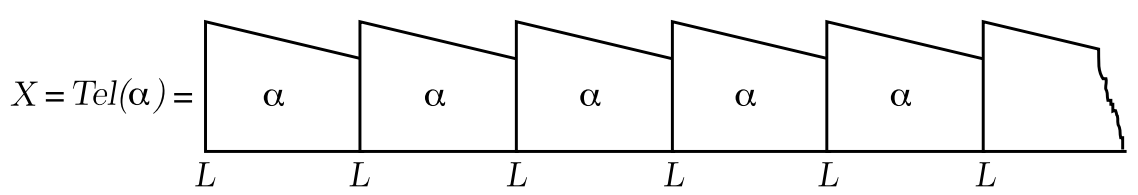

Let $d: L \rightarrow X$ be the inclusion of $L$ into the top level of the leftmost mapping cylinder of $X$ and define $s^{\prime}: X \rightarrow L$ by setting $s^{\prime}$ equal to $\alpha$ on each copy of $L$ and using the homotopies $\alpha \circ \alpha \sim \alpha$ to extend over the rest of $X$. One sees easily that $d \circ s^{\prime}$ induces the identity on the homotopy groups of $X$ and is therefore a homotopy equivalence. If $\phi$ is a homotopy inverse for $d \circ s^{\prime}$, we have $d \circ s \sim i d$, where $s=s^{\prime} \circ \phi$. This means the $d$ is a finite domination with right inverse $s$. It turns out that $[X]=(-1)^{\ell+1}[P]$.

(iii) If $X$ is dominated by a finite $C W$ complex $K$ then $\pi_{1}(X)$ is a retract of the finitely presented group $\pi_{1}(K)$, and is thus also finitely presented. The cellular chain complex $C_{*}(\widetilde{X})$ is a chain homotopy direct summand of the finite f.g. free $\mathbb{Z}\left[\pi_{1}(X)\right]$-module chain complex $\mathbb{Z}\left[\pi_{1}(X)\right] \otimes_{\mathbb{Z}\left[\pi_{1}(K)\right]} C_{*}(\widetilde{K})$, with $\widetilde{K}$ the universal cover of $K$. It follows from the algebraic theory 
of Ranicki [29] (or by the original geometric argument of Wall 40]) that $C_{*}(\tilde{X})$ is chain equivalent to a finite f.g. projective $\mathbb{Z}\left[\pi_{1}(X)\right]$-module chain complex $\mathcal{P}$.

Conversely, if $\pi_{1}(X)$ is finitely presented and $C_{*}(\tilde{X})$ is chain equivalent to a finite f.g. projective $\mathbb{Z}\left[\pi_{1}(X)\right]$-module chain complex $\mathcal{P}$ the cellular $\mathbb{Z}\left[\pi_{1}(X)\right]\left[z, z^{-1}\right]$-module chain complex of the universal cover $\widehat{X \times S^{1}}=$ $\tilde{X} \times \mathbb{R}$ of $X \times S^{1}$

$$
C_{*}\left(\widetilde{X \times S^{1}}\right)=C_{*}(\tilde{X}) \otimes_{\mathbb{Z}} C_{*}(\mathbb{R})
$$

is chain equivalent to a finite f.g. free $\mathbb{Z}\left[\pi_{1}(X)\right]\left[z, z^{-1}\right]$-module chain complex, so that $X \times S^{1}$ is homotopy equivalent to a finite $C W$ complex (by the proof of (i), using $\left.\left[X \times S^{1}\right]=0\right)$ and $X$ is finitely dominated by 1.5 .

In particular, if $\pi$ is a finitely presented group such that $\widetilde{K}_{0}(\mathbb{Z}[\pi]) \neq 0$ then there exists a finitely dominated $C W$ complex $X$ with $\pi_{1}(X)=\pi$ and such that $X$ is not homotopy equivalent to a finite $C W$ complex. See Ferry [12] for the construction of finitely dominated compact metric spaces (which are not $A N R$ 's, still less $C W$ complexes) which are not homotopy equivalent to a finite $C W$ complex.

Wall 40 obtained the finiteness obstruction of a finitely dominated $C W$ complex $X$ from $C_{*}(\tilde{X})$, using any finite f.g. projective $\mathbb{Z}\left[\pi_{1}(X)\right]$-module chain complex

$$
\mathcal{P}: \cdots \rightarrow 0 \rightarrow P_{n} \stackrel{\partial}{\rightarrow} P_{n-1} \stackrel{\partial}{\rightarrow} \cdots \stackrel{\partial}{\rightarrow} P_{1} \stackrel{\partial}{\rightarrow} P_{0} .
$$

chain equivalent to $C_{*}(\widetilde{X})$.

Definition 3.2. The projective class of $X$ is the projective class of $\mathcal{P}$

$$
[X]=\sum_{i=0}^{\infty}(-1)^{i}\left[P_{i}\right] \in K_{0}\left(\mathbb{Z}\left[\pi_{1}(X)\right]\right) .
$$

The projective class is a well-defined chain-homotopy invariant of $C_{*}(\tilde{X})$, with components

$$
[X]=(\chi(X),[X]) \in K_{0}\left(\mathbb{Z}\left[\pi_{1}(X)\right]\right)=K_{0}(\mathbb{Z}) \oplus \widetilde{K}_{0}\left(\mathbb{Z}\left[\pi_{1}(X)\right]\right),
$$

where

$$
\chi(X)=\sum_{i=0}^{\infty}(-1)^{i} \# \text { of } i \text {-cells } \in K_{0}(\mathbb{Z})=\mathbb{Z}
$$

is the Euler characteristic of $X$, and $[X]$ is the finiteness obstruction.

The instant finiteness obstruction (Ranicki [29]) of a finitely dominated $C W$ complex $X$ is a f.g. projective $\mathbb{Z}\left[\pi_{1}(X)\right]$-module $P$ representing the 
finiteness obstruction

$$
[X]=[P] \in \widetilde{K}_{0}\left(\mathbb{Z}\left[\pi_{1}(X)\right]\right)
$$

which is obtained directly from a finite domination $d: K \rightarrow X, s: X \rightarrow K$, a homotopy $h: d \circ s \simeq \operatorname{id}_{X}: X \rightarrow X$ and the cellular $\mathbb{Z}\left[\pi_{1}(X)\right]$-module chain complex $C_{*}(\widetilde{K})$ of the cover $\widetilde{K}=d^{*} \widetilde{X}$ of $K$ obtained by pullback from the universal cover $\widetilde{X}$ of $X$, namely

$$
P=\operatorname{im}\left(p: \mathbb{Z}\left[\pi_{1}(X)\right]^{n} \rightarrow \mathbb{Z}\left[\pi_{1}(X)\right]^{n}\right)
$$

with $p=p^{2}$ the projection

$$
p=\left(\begin{array}{cccc}
s \circ d & -\partial & 0 & \ldots \\
-s \circ h \circ d & 1-s \circ d & \partial & \ldots \\
s \circ h^{2} \circ d & s \circ h \circ d & s \circ d & \ldots \\
\vdots & \vdots & \vdots & \ddots
\end{array}\right)^{\infty} \quad:
$$

of a f.g. free $\mathbb{Z}\left[\pi_{1}(X)\right]$-module of rank

$$
n=\sum_{i=0}^{\infty} \# \text { of } i \text {-cells of } K \text {. }
$$

In fact, the finiteness obstruction can be obtained in this way using only the chain homotopy projection $q=s \circ d \simeq q^{2}: C_{*}(\widetilde{K}) \rightarrow C_{*}(\widetilde{K})$ induced by the homotopy idempotent $q=s \circ d \simeq q^{2}: K \rightarrow K$ (Lück and Ranicki [20.).

The finiteness obstruction has many of the usual properties of the Euler characteristic $\chi$. For instance, if $X$ is the union of finitely dominated complexes $X_{1}$ and $X_{2}$ along a common finitely dominated subcomplex $X_{0}$, then

$$
[X]=i_{1 *}\left[X_{1}\right]+i_{2 *}\left[X_{2}\right]-i_{0 *}\left[X_{0}\right]
$$

This is the sum theorem for finiteness obstructions, which was originally proved in Siebenmann's thesis [36].

The projective class of the product $X \times Y$ of finitely dominated $C W$ complexes $X, Y$ is given by

$$
[X \times Y]=[X] \otimes[Y] \in K_{0}\left(\mathbb{Z}\left[\pi_{1}(X \times Y)\right]\right)
$$

leading to the product formula of Gersten [14 for the finiteness obstruction

$$
[X \times Y]=\chi(X) \otimes[Y]+[X] \otimes \chi(Y)+[X] \otimes[Y] \in \widetilde{K}_{0}\left(\mathbb{Z}\left[\pi_{1}(X \times Y)\right]\right) .
$$

In particular, $\left[X \times S^{1}\right]=0$, giving an algebraic proof of the result (1.5) that $X \times S^{1}$ is homotopy equivalent to a finite $C W$ complex. 
A fibration $p: E \rightarrow B$ with finitely dominated fibre $F$ induces transfer maps in the projective class groups

$$
p^{!}: K_{0}\left(\mathbb{Z}\left[\pi_{1}(B)\right]\right) \rightarrow K_{0}\left(\mathbb{Z}\left[\pi_{1}(E)\right]\right) ;[X] \mapsto[Y]
$$

sending the projective class of a finitely dominated $C W$ complex $X$ with a $\pi_{1}$-isomorphism $f: X \rightarrow B$ to the projective class of the pullback $Y=$ $f^{!} E$, which is a finitely dominated $C W$ complex with a $\pi_{1}$-isomorphism $f^{!}: Y \rightarrow E$

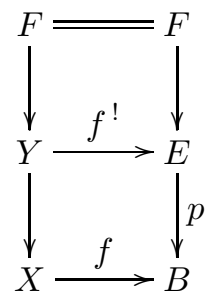

Lück 18] obtained the following algebraic description of $p^{\text {! }}$, generalizing the product formula.1 Let $\widetilde{F}$ be the pullback to $F$ of the universal cover $\widetilde{E}$ of $E$. The fibration $p$ induces a morphism of rings

$$
U: \mathbb{Z}\left[\pi_{1}(B)\right] \rightarrow H_{0}\left(\operatorname{Hom}_{\mathbb{Z}\left[\pi_{1}(E)\right]}\left(C_{*}(\widetilde{F}), C_{*}(\widetilde{F})\right)\right)^{o p}
$$

sending the homotopy class of a loop $\omega: S^{1} \rightarrow B$ to the chain homotopy class of the parallel transport chain equivalence $U(\omega): C_{*}(\widetilde{F}) \rightarrow C_{*}(\widetilde{F})$. A f. g. projective $\mathbb{Z}\left[\pi_{1}(B)\right]$-module

$$
Q=\operatorname{im}\left(q: \mathbb{Z}\left[\pi_{1}(B)\right]^{n} \rightarrow \mathbb{Z}\left[\pi_{1}(B)\right]^{n}\right) \quad\left(q=q^{2}\right)
$$

induces a $\mathbb{Z}\left[\pi_{1}(E)\right]$-module chain complex

$$
Q^{!}=\mathcal{C}\left(U(q): C_{*}(\widetilde{F})^{n} \rightarrow C_{*}(\widetilde{F})^{n}\right) \quad\left(U(q) \simeq U(q)^{2}\right)
$$

which is algebraically finitely dominated, i.e. chain equivalent to a finite f. g. projective chain complex. The transfer map is given algebraically by

$$
p^{!}[Q]=\left[Q^{!}\right] \in K_{0}\left(\mathbb{Z}\left[\pi_{1}(E)\right]\right) .
$$

\section{The TOPOlOGiCAL SPACE-FORM PROBLEM}

Another problem in which a finiteness obstruction arises is the topological space-form problem. This is the problem of determining which groups can act freely and properly discontinuously on $S^{n}$ for some $n$.

Swan, [37, solved a homotopy version of this problem by proving that a finite group $G$ of order $n$ which has periodic cohomology of period $q$ acts freely on a finite complex of dimension $d q-1$ which is homotopy equivalent

\footnotetext{
${ }^{1}$ See Lück and Ranicki 19 for the algebraic transfer map in the surgery obstruction groups.
} 
to a $(d q-1)$-sphere. Here, $d$ is the greatest common divisor of $n$ and $\phi(n)$, $\phi$ being Euler's $\phi$-function.

One might ask whether such a $G$ can act on $S^{q-1}$, but this refinement leads to a finiteness obstruction. It follows from Swan's argument that $G$ acts freely on a countable $q$-1-dimensional complex $X$ homotopy equivalent to $S^{q-1}$ and that $X / G$ is finitely dominated. The finiteness obstruction of $X / G$ need not be zero, however, so not every group with cohomology of period $q$ can act freely on a finite complex homotopy equivalent to $S^{q-1}$. Algebraically, the point is that finite groups with $q$-periodic cohomology have $q$-periodic resolutions by f. g. projective modules but need not have $q$-periodic resolutions by f. g. free modules.

After a great deal of work involving both the finiteness obstruction and surgery theory, see Madsen, Thomas and Wall [21, it turned out that a group $G$ acts freely on $S^{n}$ for some $n$ if and only if all of its subgroups of order $p^{2}$ and $2 p$ are cyclic (the condition of Milnor). This is in contrast to the linear case. A group $G$ acts linearly on $S^{n}$ for some $n$ if and only if all subgroups of order $p q, p$ and $q$ not necessarily distinct primes, are cyclic. See Davis and Milgram [9] for a book-length treatment, and Weinberger 41], p. 110, for a brief discussion.

\section{The Siebenmann end obstruction}

The most significant application of the finiteness obstruction to the topology of manifolds is via the end obstruction.

An end $\epsilon$ of an open $n$-dimensional manifold $W$ is tame if there exists a sequence $W \supset U_{1} \supset U_{2} \supset \ldots$ of finitely dominated neighbourhoods of $\epsilon$ with

$$
\bigcap_{i} U_{i}=\emptyset, \pi_{1}\left(U_{1}\right) \cong \pi_{1}\left(U_{2}\right) \cong \cdots \cong \pi_{1}(\epsilon) .
$$

The end is collared if there exists a neighbourhood of the type $M \times[0, \infty)$ for some closed $(n-1)$-dimensional manifold $M$, i.e. if $\epsilon$ is the interior of a compactification $W \cup M$ with boundary component $M$.

Theorem 5.1. (Siebenmann [36]) A tame end $\epsilon$ of an open $n$-dimensional manifold $W$ has an end obstruction

$$
[\epsilon]=\lim _{i}\left[U_{i}\right] \in \widetilde{K}_{0}\left(\mathbb{Z}\left[\pi_{1}(\epsilon)\right]\right)
$$

such that $[\epsilon]=0$ if (and for $n \geq 6$ only if) $\epsilon$ can be collared.

Novikov's 1965 proof of the topological invariance of the rational Pontrjagin classes made use of the end obstruction in the unobstructed case when $\pi$ is a free abelian group. The subsequent work of Lashof, Rothenberg, Casson, Sullivan, Kirby and Siebenmann on the Hauptvermutung for high-dimensional manifolds made overt use of the end obstruction ([33]). 
See sections 7 and 8 below for brief accounts of the applications of the end obstruction to splitting theorems and triangulation of high-dimensional manifolds.

See Chapman [7], Quinn [27], [28] and Connolly and Vajiac [8] for the controlled end obstruction, and Ranicki [32] for the bounded end obstruction.

See Hughes and Ranicki 15 for a book-length treatment of ends and the end obstruction.

\section{Connections with Whitehead torsion}

The finiteness obstruction deals with the existence of a finite $C W$ complex $K$ in a homotopy type, while Whitehead torsion deals with the uniqueness of $K$. There are many deep connections between the finiteness obstruction and Whitehead torsion, which on the purely algebraic level correspond to the connections between the algebraic $K$-groups $K_{0}, K_{1}$ (or rather $\widetilde{K}_{0}$, $W h)$.

The splitting theorem of Bass, Heller and Swan [2]

$$
W h(\pi \times \mathbb{Z})=W h(\pi) \oplus \widetilde{K}_{0}(\mathbb{Z}[\pi]) \oplus \widetilde{\operatorname{Nil}_{0}}(\mathbb{Z}[\pi]) \oplus \widetilde{\operatorname{Nil}_{0}}(\mathbb{Z}[\pi])
$$

involves a split injection

$$
\widetilde{K}_{0}(\mathbb{Z}[\pi]) \rightarrow W h(\pi \times \mathbb{Z}) ;[P] \mapsto \tau\left(z: P\left[z, z^{-1}\right] \rightarrow P\left[z, z^{-1}\right]\right) .
$$

If $X$ is a finitely dominated space then 1.5 gives a homotopy equivalence $\phi: X \times S^{1} \rightarrow K$ to a finite $C W$ complex $K$, uniquely up to simple homotopy equivalence. Ferry $\left[13\right.$ identified the finiteness obstruction $[X] \in \widetilde{K}_{0}(\mathbb{Z}[\pi])$ $\left(\pi=\pi_{1}(X)\right)$ with the Whitehead torsion $\tau(f) \in W h(\pi \times \mathbb{Z})$ of the composite self homotopy equivalence of a finite $C W$ complex

$$
f: K \stackrel{\phi^{-1}}{\longrightarrow} X \times S^{1} \stackrel{\operatorname{id}_{X \times-1}}{\longrightarrow} X \times S^{1} \stackrel{\phi}{\longrightarrow} K .
$$

In Ranicki [30, 31] it was shown that $[X] \mapsto \tau(f)$ corresponds to the split injection

$$
\widetilde{K}_{0}(\mathbb{Z}[\pi]) \rightarrow W h(\pi \times \mathbb{Z}) ;[P] \mapsto \tau\left(-z: P\left[z, z^{-1}\right] \rightarrow P\left[z, z^{-1}\right]\right)
$$

which is different from the original split injection $(*)$ of [2].

\section{The SPLitTing OBSTRUCTION}

The finiteness obstruction arises in most classification problems in highdimensional topology. Loosely speaking, proving that two manifolds are homeomorphic involves decomposing them into homeomorphic pieces. The finiteness obstruction is part of the obstruction to splitting a manifold into pieces. The nonsimply-connected version of Browder's $M \times \mathbb{R}$ Theorem is 
a case in point. In [5], Browder proved that if $M^{n}, n \geq 6$, is a $P L$ manifold without boundary, $f: M \rightarrow K \times \mathbb{R}^{1}$ is a $(P L)$ proper homotopy equivalence, and $K$ is a simply-connected finite complex, then $M$ is homeomorphic to $N \times \mathbb{R}^{1}$ for some closed manifold $N$ homotopy equivalent to $K$.

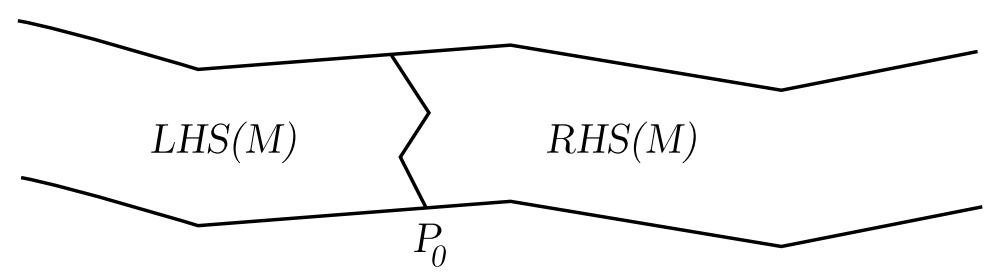

When $K$ is connected but not simply-connected, a finiteness obstruction arises. Here is a quick sketch of the argument: It is not difficult to show that $M$ is 2 -ended. The proper homotopy equivalence $f: M \rightarrow K \times$ $\mathbb{R}^{1}$ gives us a proper $P L$ map $p: M \rightarrow \mathbb{R}$. If $c \in \mathbb{R}$ is not the image of any vertex, then $p^{-1}(c)$ is a bicollared $P L$ submanifold of $M$ which separates the ends. Connected summing components along arcs allows us to assume that $P_{0}=p^{-1}(c)$ is connected and a disk-trading argument similar to one in Browder's paper allows us to assume that $\pi_{1} P_{0} \rightarrow \pi_{1} M$ is an isomorphism. See Siebenmann [36] for details. An application of the recognition criterion discussed in the third paragraph of this paper shows that the two components of $M-P_{0}$, which we denote by $R H S(M)$ and $L H S(M)$, respectively, are finitely dominated. By the sum theorem,

$$
[R H S(M)]+[L H S(M)]=0 \in \widetilde{K}_{0}\left(\mathbb{Z}\left[\pi_{1}(M)\right]\right) .
$$

It turns out that the vanishing of $[R H S(M)]=-[L H S(M)]$ is necessary and sufficient for $M$ to be homeomorphic to a product $N \times \mathbb{R}$, provided that $\operatorname{dim}(M) \geq 6$. This is one of the main results of [36].

It is possible to realize the finiteness obstruction $\sigma \in \widetilde{K}_{0}\left(\mathbb{Z}\left[\pi_{1}(K)\right]\right)$ for an $n$-dimensional manifold $M^{n}$ proper homotopy equivalent to $K \times \mathbb{R}$ for some finite $K$ whenever $\sigma+(-1)^{n-1} \sigma^{*}=0$ and $n \geq 6$. If we only require that $M$ be properly dominated by some $K \times \mathbb{R}$, then any finiteness obstruction $\sigma$ can be realized (cf. Pedersen and Ranicki [26].) A similar obstruction arises in the problem of determining whether a map $p: M^{n} \rightarrow S^{1}$ is homotopic to the projection map of a fiber bundle (Farrell [10]).

The geometric splitting of two-ended open manifolds into right and left sides is closely related to the proof of the algebraic splitting theorem of Bass, Heller and Swan [2] for $W h(\pi \times \mathbb{Z})$ - see Ranicki [32]. 


\section{THE TRIANGULATION OF MANIFOLDS}

The finiteness obstruction arises in connection with another of the fundamental problems of topology: Is every compact topological manifold without boundary homeomorphic to a finite polyhedron? We will examine this problem in much greater detail.

The triangulation problem was solved affirmatively for two-dimensional manifolds by Rado in 1924 and for three-dimensional manifolds by Moise in 1952. Higher dimensions proved less tractable, [ a circumstance which encouraged the formulation of weaker questions such as the following homotopy triangulation problem: Does every compact topological manifold have the homotopy type of some finite polyhedron?

The first solution of this problem came as a corollary to Kirby and Siebenmann's theory of $P L$ triangulations of high-dimensional topological manifolds. By a theorem of Hirsch, every topological manifold $M^{n}$ has a well-defined stable topological normal disk bundle. The total space of this bundle is a closed neighborhood of $M$ in some high-dimensional euclidean space. In [16], Kirby and Siebenmann proved that a topological $n$-manifold, $n \geq 6$, has a $P L$ structure if and only if this stable normal bundle reduces from TOP to $P L$. As an immediate corollary, they deduced that every compact topological manifold has the homotopy type of a finite polyhedron, since each $M$ is homotopy equivalent to the total space of the unit disk bundle of its normal disk bundle and the total space of the normal disk bundle is a $P L$ manifold because its normal bundle is trivial. The argument of Kirby and Siebenmann also shows that each compact topological manifold has a well-defined simple homotopy type. A more refined argument, see p.104 of Kirby and Siebenmann [17], shows that every closed topological manifold of dimension $\geq 6$ is a $T O P$ handlebody. From this it follows immediately that every compact topological manifold is homotopy equivalent to a finite $C W$ complex and therefore to a finite polyhedron.

This positive solution to the homotopy-triangulation problem suggests that we should look for large naturally-occurring classes of compact topological spaces which have the homotopy types of finite polyhedra. In 1954, K. Borsuk [- [- conjectured that every compact metrizable $A N R$ should have the homotopy type of a finite polyhedron. This became widely known as Borsuk's Conjecture.

\footnotetext{
${ }^{2}$ In fact, Casson has shown that there are compact four-manifolds without boundary which are not homeomorphic to finite polyhedra (Akbulut and McCarthy 《్, p.xvi). The question is still open in dimensions greater than or equal to five.
} 
The Borsuk Conjecture was solved by J. E. West, [42], using results of T. A. Chapman, which, in turn, were based on an infinite-dimensional version of Kirby-Siebenmann's handle-straightening argument. In a nutshell, Chapman proved that every compact manifold modeled on the Hilbert cube $\left(\equiv \prod_{i=1}^{\infty}[0,1]\right)$ is homotopy equivalent to a finite complex and West showed that every compact $A N R$ [ $^{\beta}$ is homotopy equivalent to a compact manifold modeled on the Hilbert cube. A rather short finite-dimensional proof of the topological invariance of Whitehead torsion, together with the Borsuk Conjecture was given by Chapman in [6]. See Ranicki and Yamasaki [34] for a more recent proof, which makes use of controlled algebraic $K$-theory.

\section{REFERENCES}

[1] S. Akbulut and J. D. McCarthy, Casson's invariant for oriented homology 3spheres, Mathematical Notes 36, Princeton University Press, 1990.

[2] H. Bass, A. Heller, and R. Swan, The Whitehead group of a polynomial extension, Publ. Math. IHES 22 (1964), 61-80.

[3] K. Borsuk, Zur kombinatorischen Eigenschaften der Retrakte, Fund. Math. 21 (1933), 91-98.

[4] Sur l'élimination de phenomènes paradoxaux en topologie générale, Proc. Internat. Congr. Math., Amsterdam, 1954, pp. 197-208.

[5] W. Browder, Structures on $M \times R$, Proc. Cam. Phil. Soc. 61 (1965), 337-345.

[6] T. A. Chapman, Invariance of torsion and the Borsuk conjecture, Canad. J. Math. 32 (1980), 1333-1341.

[7] — Controlled simple homotopy theory and applications, Lecture Notes in Mathematics 1009, Springer, 1983.

[8] F. X. Connolly and B. Vajiac, An end theorem for stratified spaces, Invent. Math. 135 (1999), 519-543.

[9] J. Davis and J. Milgram, A survey of the spherical space form problem, Mathematical Reports 2, Harwood, 1984.

[10] F. T. Farrell, The obstruction to fibering a manifold over a circle, Indiana U. Math. J. 21 (1971), 315-346.

[11] _ and L. E. Jones, Classical aspherical manifolds, CBMS Regional Conference Series in Mathematics 75 (1990), AMS.

[12] S. Ferry, Homotopy, simple homotopy, and compacta, Topology 19 (1980), 101-110.

[13] _ A simple homotopy approach to the finiteness obstruction, Shape theory, Dubrovnik 1981, Lecture Notes in Mathematics 870, Springer, 1981, pp. 73-81.

[14] S. Gersten, A product formula for Wall's obstruction, Amer. J. Math. 88 (1966), 337-346.

[15] B. Hughes and A. Ranicki, Ends of complexes, Cambridge Tracts in Mathematics 123, Cambridge University Press, 1996.

[16] R. C. Kirby and L. C. Siebenmann, On the triangulation of manifolds and the Hauptvermutung, Bull. AMS 75 (1969), 742-749.

[17] _ and Foundational essays on topological manifolds, smoothings, and triangulations, Ann. of Maths. Study 88, Princeton University Press, 1977.

[18] W. Lück, The transfer maps induced in the algebraic $K_{0}$ - and $K_{1}$-groups by a fibration I., Math. Scand. 59 (1986), 93-121.

\footnotetext{
${ }^{3}$ A compact metrizable space $X$ is an $A N R$ if and only if it embeds as a neighborhood retract in separable Hilbert space. If $X$ has finite covering dimension $\leq n$, separable Hilbert space can be replaced by $\mathbb{R}^{2 n+1}$.
} 
[19] — and A. Ranicki, Surgery transfer, Algebraic Topology, Göttingen, 1984, Lecture Notes in Mathematics 1361, Springer, 1985, pp. 167-246.

[20] — and Chain homotopy projections, J. of Algebra 120 (1989), 361391.

[21] I. Madsen, C. B. Thomas, and C. T. C. Wall, The topological spherical space form problem II, Topology 15 (1976), 375-382.

[22] M. Mather, Counting homotopy types of topological manifolds, Topology 4 (1965), 93-94.

[23] J. Milnor, On spaces having the homotopy type of a $C W$ complex, Trans. A.M.S. 90 (1959), 272-280.

[24] G. Mislin, Wall's finiteness obstruction, Handbook of algebraic topology, Elsevier, 1995, pp. 1259-1291.

[25] R. Oliver, Projective class groups of integral group rings: a survey, Orders and their applications (Oberwolfach, 1984), Lecture Notes in Mathematics 1142, Springer, 1985, pp. 211-232

[26] E. Pedersen and A. Ranicki, Projective surgery theory, Topology 19 (1980), 239254.

[27] F. Quinn, Ends of maps I., Ann. of Math. 110 (1979), 275-331.

[28] _ Ends of maps II., Invent. Math. 68 (1982), 353-424.

[29] A. Ranicki, The algebraic theory of finiteness obstruction, Math. Scand. 57 (1985), 105-126.

[30] Algebraic and geometric splittings of the $K$ - and L-groups of polynomial extensions, Proc. 1985 Poznań Topology Conf., Lecture Notes in Mathematics 1217, Springer, 1986, pp. 321-363.

[31] - Algebraic theory of torsion II. Products, K-theory 1 (1987), 115-170.

[32] _ Lower $K$ - and L-theory, LMS Lecture Notes 178, Cambridge, 1992.

[33] _ (ed.), The Hauptvermutung Book, K-Monographs in Mathematics 1, Kluwer, 1996.

[34] _ and M. Yamasaki, Controlled K-theory, Topology and Its Applications 61 (1995), 1-59.

[35] J. Rosenberg, Algebraic K-theory and Its Applications, Graduate Texts in Mathematics 147, Springer, 1994.

[36] L. C. Siebenmann, Finding a boundary for an open manifold of dimension $\geq 5$, Ph.D. thesis, Princeton University, 1965.

[37] R. G. Swan, Periodic resolutions for finite groups, Ann. of Math. 72 (1960), 267291.

[38] K. Varadarajan, The finiteness obstruction of C.T.C. Wall, J. Wiley, 1989.

[39] C. T. C. Wall, Finiteness conditions for CW complexes, Ann. of Math. 81 (1965), $55-69$.

[40] (1966), 129-139.

[41] S. Weinberger, The topological classification of stratified spaces, U. of Chicago Press, 1994.

[42] J. E. West, Mapping Hilbert cube manifolds to ANR's: A solution to a conjecture of Borsuk, Ann. of Math. 106 (1977), 1-18. 
S.F.: Dept. of Mathematics, Rutgers University, New Brunswick, NJ 08903, USA

E-mail address: sferry@math.rutgers.edu

A.R.: Dept. of Mathematics and Statistics, University of Edinburgh, EdinBURGH EH9 3JZ, SCOTLAND, UK

E-mail address: aar@maths.ed.ac.uk 